\title{
Effect of Multidisciplinary Case Conferences on Physician Decision Making: Breast Diagnostic Rounds
}

\author{
Tianne J. Foster ${ }^{1}$, Antoine Bouchard-Fortier ${ }^{1}$, Ivo A. Olivotto ${ }^{2}$, May Lynn Quan ${ }^{1}$ \\ 1. Surgical Oncology, University of Calgary/Tom Baker Cancer Center 2. Department of Oncology, \\ University of Calgary/Tom Baker Cancer Center
}

$\square$ Corresponding author: Tianne J. Foster, tianne.foster2@ucalgary.ca

Disclosures can be found in Additional Information at the end of the article

\section{Abstract}

Purpose: To evaluate the utility of multidisciplinary case conferences (MCCs) on physician decision making in benign and malignant breast disease management.

Methods: Patients with interesting or challenging diagnostic or management issues were discussed at biweekly diagnostic breast MCCs. Prior to discussion, a clinical summary and intended management plan prior to the MCC was presented. For each case, diagnostic images/histopathology were centrally reviewed after which group discussion achieved a management consensus which was documented prospectively. Initial management plans were compared to the post-MCC consensus. A change in a management plan was defined as a consensus plan different from the pre-MCC plan or no definite plan prior to the MCC.

Results: From November 2014 to December 2015, 76 patients (43 malignant and 33 benign diagnoses) were discussed in 19 MCCs. All cases presented resulted in a consensus management recommendation. Thirty-one case discussions (41\%) resulted in a changed management plan (20 malignant and 11 benign diagnoses). Management changes included avoidance of immediate surgery ( $9 \%$ of cases), change in the type of surgery (5\%), non-invasive investigation to invasive/surgical intervention (7\%), and detection of a new suspicious lesion (1\%).

Conclusion: MCCs had a substantial impact on physician decision making. Management plans changed in $41 \%$ of cases presented, the majority due to new/clarified diagnostic information. Presentation of cases at MCCs should be encouraged, especially for challenging diagnostic or management issues regarding malignant or benign breast diagnoses.

Received 10/20/2016

Review began 10/28/2016

Review ended 11/21/2016

Published 11/24/2016

C) Copyright 2016

Foster et al. This is an open access article distributed under the terms of the Creative Commons Attribution License CC-BY 3.0., which permits unrestricted use, distribution, and reproduction in any medium, provided the original author and source are credited.
Categories: Radiation Oncology, General Surgery, Oncology

Keywords: multidisciplinary, case conference, tumour board, breast cancer, decision-making

\section{Introduction}

Breast disease management is complex and multidisciplinary. An effective diagnosis is based on clinical-radiology-pathology correlation which depends on regular open communication between disciplines [1]. Cancer Care Ontario (CCO) proposed standards for multidisciplinary case conferences (MCCs) and described a framework including recognition of primary and secondary functions. The primary function of an MCC is to ensure all diagnostic tests and treatment options are considered for individual patients [2]. Secondary functions include 
continuing education to aid in appropriate and timely referrals, contribute to research, and ensure quality care [2].

In Calgary, Alberta, breast diagnostic MCCs were instituted bi-weekly in 2010 to review cases with benign or malignant breast pathology with challenging diagnostic or management issues. Many studies have been conducted on the impact of MCCs for patients with malignant breast pathology. However, to our knowledge, no studies have assessed the value of MCCs for patients with both benign and malignant pathology. This is a report of a prospective evaluation of the utility of MCCs on physician decision making for patients with benign and malignant breast diseases.

\section{Materials And Methods}

A prospective evaluation was conducted of MCCs that occurred every second Wednesday afternoon for one hour, from November 5th, 2014 to December 9th, 2015 but excluding July to September 2015. The MCCs were video-linked between six sites in the city of Calgary, Alberta, Canada. Patients with diagnostic or management issues were selected for presentation by the attending physician, generally their surgeon. The cases included benign or malignant pathology and may have been new diagnoses or recurrent disease. MCC attendees included surgeons (general surgeons and surgical oncologists), subspecialty trained radiologists and pathologists, medical and radiation oncologists, general practitioners with an oncology focus, nurse coordinators, and trainees. A summary of the patient's clinical history was presented and the question(s) for the MCC were described by the presenting physician who also stated his/her intended management if the MCC had not been available. Diagnostic images and histopathology were reviewed by a breast expert radiologist and pathologist, respectively, so that all cases had a 'central' review. The pre-MCC intended management was recorded and compared to the MCC consensus management recommendation. A management "change" was defined as a difference compared to the pre-MCC plan or if there was no definite management plan prior to the MCC.

\section{Results}

Nineteen biweekly MCCs occurred during which 76 patients were discussed. One to eight patients (median $=4$ ) were discussed at each MCC. A minimum of one radiologist, pathologist, and surgeon were present at each MCC. Radiation and medical oncologist attendance was variable, but there were complementary weekly city-wide oncology management rounds at which patients' oncologic management may have been discussed. The range of participants at any given round was 5-15. Trainee and non-physician attendance was not recorded. Attendance was recorded mainly for the purposes of continuing medical education credit, and thus attendance from video-linked sites was not well captured.

Of the 76 cases, 33 had only benign pathology, and 43 had malignant diagnoses. Five benign cases had a prior history of breast cancer. After review of the diagnostic information and MCC discussion, management changes were recommended for 31 (41\%) patients (Table 1). Sixty-two of the cases (82\%) had a proposed plan prior to the MCC; whereas for 14 (18\%), the management decision was dependent on the discussion. Of the 31 cases with a management plan change, 20 had malignant diagnoses (65\%), and 11 were benign (35\%). Fourteen cases (45\%) changed due to new or clarified information from diagnostic imaging, 9 cases (29\%) changed due to new or clarified details from histopathology, and 8 cases (26\%) changed after both diagnostic imaging and histopathology review and group discussion. 


\section{Cureus}

BCS

BCS

BCS

Biopsy right lesion

Follow /no excision

Follow

Image localization

No SLNB

Plan dependent on MCC consensus

Plan dependent on MCC consensus

Plan dependent on MCC consensus

Plan dependent on MCC consensus

Plan dependent on MCC consensus

Plan dependent on MCC consensus

Plan dependent on MCC consensus

Plan dependent on MCC consensus

Radiotherapy

Refer for surgery

Refer for surgery

Segmental mastectomy and SLNB

SLNB and ?needle core or?

excisional biopsy

Surgical excision

Surgical excision
Mastectomy

Biopsy

MRI, neoadjuvant chemotherapy +

Herceptin

Biopsy right lesion and suspicious left lesion

Surgical excision

Re-biopsy

Major duct excision, no localization

Perform SLNB

Biopsy

BCS

Mastectomy

Surgical excision

Re-excision of margins

No biopsy/follow

Further imaging

Radiotherapy

Biopsy/external histopathology slide review

Repeat biopsy

Radiotherapy

Re-imaging with CT

Excisional biopsy, no SLNB

No surgical excision

Vacuum assisted biopsy

$$
\text { Malignant (3) }
$$

3

Malignant

1

$\begin{array}{ll}\text { Malignant } & 1\end{array}$

Malignant 1

$\begin{array}{ll}\text { Benign } & 1\end{array}$

Benign 1

$\begin{array}{ll}\text { Benign } & 1\end{array}$

Malignant 1

Malignant (1) Benign (1) 2

$\begin{array}{ll}\text { Malignant } & 1\end{array}$

Malignant $\quad 1$

Malignant (2) $\quad 2$

Malignant 1

Benign (2) 2

Benign (3) 3

Malignant (2) $\quad 2$

$\begin{array}{ll}\text { Benign } & 1\end{array}$

Malignant 1

$\begin{array}{ll}\text { Malignant } & 1\end{array}$

$\begin{array}{ll}\text { Malignant } & 1\end{array}$

$\begin{array}{ll}\text { Malignant } & 1\end{array}$

$\begin{array}{ll}\text { Malignant } & 1\end{array}$

Benign 1

\section{TABLE 1: Changes in Management as a Result of a Review of Diagnostic Imaging}

Films/Histopathology and Discussion

Abbreviations: SLNB - sentinel lymph node biopsy, CT - computed tomography, BCS - breast conserving surgery 


\section{Cureus}

The changes that took place involved avoidance of immediate surgery in 7/31 (23\%) cases, change in the type of surgery 4/31 (13\%), non-invasive investigation to invasive/surgical intervention 5/31 (16\%), and detection of a new suspicious lesion in one case (3\%) (Table 2). Our results demonstrated a $9 \%$ avoidance of surgery after MCC discussion. In a lower percentage of cases (7\%), the post-MCC plan determined that surgical/invasive intervention was necessary as opposed to the pre-MCC intent for non-invasive investigation (Table 2). In those five cases, a review of both diagnostic imaging and histopathology determined that a sentinel lymph node biopsy (SLNB) should be provided, and a second patient should be rebiopsied. Review of histology with discussion resulted in a recommendation for surgical excision; a biopsy was recommended instead of a previously planned radiotherapy, and in one case, imaging review with discussion resulted in a plan for surgical excision. In $5 \%$ of the cases, the type of surgery changed; three cases changed from breast conserving surgery to mastectomy, and one case recommended a sentinel lymph node biopsy (SLNB) not be performed. In one case, a new suspicious lesion was revealed for which a biopsy was recommended (Table 2).

\begin{tabular}{|c|c|c|c|}
\hline Post-MCC consensus plan & $\begin{array}{l}\text { No. of } \\
\text { cases }\end{array}$ & $\begin{array}{l}\text { Percent of total } \\
\text { cases (\%) }\end{array}$ & Based on review/discussion of \\
\hline No immediate surgery & 7 & 9.2 & Imaging alone (5) Histopathology and Imaging (2) \\
\hline Change in type of surgery & 4 & 5.2 & Imaging alone (3) Histopathology and Imaging (1) \\
\hline $\begin{array}{l}\text { Surgical/invasive intervention } \\
\text { recommended }\end{array}$ & b & 6.6 & $\begin{array}{l}\text { Imaging alone (1) HIstopathology alone (2) } \\
\text { Histopathology and Imaging (2) }\end{array}$ \\
\hline Biopsy new suspicious lesion & 1 & 1.3 & Imaging alone \\
\hline
\end{tabular}

TABLE 2: Overall Changes in Management Plans after Multidisciplinary Conference Case Reviews

\section{Discussion}

Of the 76 cases evaluated as part of this prospective study, a change in management recommendation occurred in $41 \%$ following multidisciplinary discussion. By presenting selected, challenging cases rather than all consecutive new diagnoses, there may have been a greater likelihood for management plan changes. However, even when individual patient management plans did not change, others have reported a benefit from MCCs regarding validation and building consensus about management of future patients [3-5].

In the United States, breast centers have been established since 1979 conveying an importance for multidisciplinary care in breast disease. Many breast centers conform to the standards set forth by the National Accreditation Program for Breast Centers [6-7]. Similarly in Europe, the European Accreditation of Breast Units section within the European Society of Mastology provides guidelines for breast centers in Europe [6]. On both sides of the Atlantic, conducting regular, interdisciplinary cancer case conferences is one of the standards required for a breast center to maintain accreditation $[6,8]$. Uniform Canadian national standards may be a valuable resource.

In Canada, the use of MCCs and their structure is variable between centers. In 2006, Cancer 
Care Ontario made recommendations regarding the structure, function, and purpose of MCCs. In 2008, minimum criteria were established for Ontario sites conducting MCCs [2, 9]. Any site treating more than 35 cancer patients within a specific disease site was required to hold an MCC in the specific subspecialty [2]. Cancer Care Ontario also established guidelines for the meeting format, team members and attendees, their respective roles and responsibilities, and institutional requirements such as video conferencing and teleconferencing capabilities [2]. Other jurisdictions do not have published specific standards for MCCs.

MCCs have been shown to impact diagnosis, patient care planning, and compliance with clinical practice guidelines [10-14]. A systematic review of 27 studies published from 1995 to 2015 demonstrated management plan changes in $19 \%$ to $34.5 \%$ of cases discussed in oncology MCCs [3, 10]. Studies with a retrospective design reported a higher incidence of changes (52\%) $[3,15]$. In the current study, a change rate of $41 \%$ is comparable to other prospective studies [3].

During the MCC review, physicians discussed concordance of findings between histopathology and diagnostic imaging while determining their treatment plan. Much of the discussion involved clarification of the current imaging or histopathology and may not have resulted in a specific change to the report itself. In a recent study, Prakash et al., [1] described the outcomes of weekly, multidisciplinary breast radiology-histopathology correlation conferences for percutaneous breast core needle biopsies which resulted in a change in management to avoid surgery in $2.1 \%$ of cases (29/1387) and detected additional cancers at a rate of 2.2 per 1000 cases [1]. Improved concordance after core biopsy review for benign cases has also been demonstrated [16]. Our review demonstrated that presentation at the MCC helped avoid surgery in $9 \%$ of the cases and found a new lesion in one patient (1.3\%).

Many studies have found that MCCs positively impact patient care, and a few have shown survival benefits associated with MCC presentation [17-22]. A study from the United Kingdom retrospectively compared patient outcomes and breast cancer survival over five years in one health board without MCCs to centers in a health board which introduced an MCC review process. It was found that breast cancer mortality was $18 \%$ lower in the health board implementing MCCs as compared to the health board without MCCs [17]. It was also found that within the health board implementing MCCs, there was less variation in breast cancer survival rates between hospitals [17].

Regardless of these benefits, some still question the efficiency and impact of MCCs [5, 23]. In 2015, the American Society of Clinical Oncology (ASCO) conducted an international survey of a cohort of ASCO members located outside of the United States regarding multidisciplinary cancer conferences at their centers [5]. Four hundred nine physicians responded with their reasons for attending MCCs, which included obtaining treatment recommendations (89\% of respondents) or participating in discussions (86\%). However, in these scenarios it was common for all patients with a new diagnosis of breast cancer to be presented at the meeting (49\% of respondents); whereas $34.5 \%$ of respondents attended MCCs where only selected new breast cancer cases were presented, and $16.5 \%$ of respondents stated that there were no selection criteria for the discussed breast cancer cases [5]. Overall, the case discussions at multidisciplinary meetings were geared toward cancer diagnoses. To date, there have not been any studies aside from correlation meetings, to our knowledge, which have evaluated the performance and utility of MCCs that included benign and malignant breast disease management. Presenting benign breast disease cases was useful in the present study as over a third of such cases had a management change.

Attendance, another common issue for MCCs, is variable although many physicians report that they believe MCCs benefit patients [5, 24-25]. In the current study, the majority of cases were presented by surgeons $77 \%$ (24/76), with minimal attendance from medical oncology who 
presented one case. Other cases were presented by radiation oncology, pathology, and a general practitioner with an oncology focus. Increased participation and case presentation by all breast cancer specialists should be encouraged.

In order to improve attendance and MCC function, many have desired for an effective moderator and improved time management $[2-3,5,23,25]$. When time is scarce among busy physicians, MCCs may be attended sporadically by those who do not find the meetings well organized and effectively time managed. Although not formally assessed, presenting select cases non-consecutively as opposed to a consecutive presentation of all breast cancer cases was perceived to allow more effective management of meeting times [25]. A limitation of our study was that the impact of the changes to the management plans on patient outcomes was not able to be determined.

MCC discussion not only benefited patients but also incited discussion of policies and care processes. This improved interdisciplinary communication. For example, initially the practice of pathologists was to uniformly recommend excision of certain benign lesions (i.e., atypical ductal hyperplasia or papilloma) found on core biopsy within the body of the pathology report. This practice was restrictive, and given the multiple discussions during MCCs on whether or not to excise such lesions, it was agreed by the group that changing the wording on the pathology reports to "recommendation for surgical consultation" was preferred. This improved interdisciplinary communication among participants in MCCs has been noted as a benefit of multidisciplinary meetings [26-27].

\section{Conclusions}

This study demonstrated that multidisciplinary case conferences had a substantial impact on physician decision making for both benign and malignant breast disease. Nearly half of the case discussions (31 of 76) resulted in a change in the clinical recommendation. The majority of management changes were based on new/clarified diagnostic imaging or histopathology information. Presentation of cases at MCCs should be encouraged, especially for challenging diagnostic or management issues.

\section{Additional Information}

\section{Disclosures}

Human subjects: All authors have confirmed that this study did not involve human participants or tissue. Animal subjects: All authors have confirmed that this study did not involve animal subjects or tissue. Conflicts of interest: In compliance with the ICMJE uniform disclosure form, all authors declare the following: Payment/services info: All authors have declared that no financial support was received from any organization for the submitted work. Financial relationships: All authors have declared that they have no financial relationships at present or within the previous three years with any organizations that might have an interest in the submitted work. Other relationships: All authors have declared that there are no other relationships or activities that could appear to have influenced the submitted work.

\section{References}

1. Prakash S, Venkataraman S, Slanetz PJ, et al.: Improving patient care by incorporation of multidisciplinary breast radiology-Pathology Correlation Conference. Can Assoc Radiol J. 2016, 67:122-129. 10.1016/j.carj.2015.07.003

2. Wright FC, De Vito C, Langer B, Hunter A, et al.: Multidisciplinary cancer conferences: a systematic review and development of practice standards. Eur J Cancer. 2007, 43:1002-1010. 10.1016/j.ejca.2007.01.025

3. Pillay B, Wootten AC, Crowe $\mathrm{H}$, et al.: The impact of multidisciplinary team meetings on 
patient assessment, management and outcomes in oncology settings: a systematic review of the literature. Cancer Treat Rev. 2016, 42:56-72. 10.1016/j.ctrv.2015.11.007

4. Rao K, Manya K, Azad A, et al.: Uro-oncology multidisciplinary meetings at an Australian tertiary referral centre--impact on clinical decision-making and implications for patient inclusion. BJU Int. 2014, 114 Suppl 1:50-54. 10.1111/bju.12764

5. El Saghir NS, Charara RN, Kreidieh FY, et al.: Global practice and efficiency of multidisciplinary tumor boards: results of an American Society of Clinical Oncology international survey. J Glob Oncol. 2015, 1:57-64. 10.1200/jgo.2015.000158

6. Bensenhaver J, Winchester DP: Surgical leadership and standardization of multidisciplinary breast cancer care: the evolution of National Accreditation Program for Breast Centers. Surg Oncol Clin N Am. 2014, 23:609-616. 10.1016/j.soc.2014.03.005

7. Winchester DP: The national accreditation program for breast centers: a multidisciplinary approach to improve the quality of care for patients with diseases of the breast. Breast J. 2008, 14:409-411. 10.1111/j.1524-4741.2008.00639.x

8. EUSOMA: The requirements of a specialist breast unit . Eur J Cancer. 2000, 36:2288-2293. 10.1016/S0959-8049(00)00180-5

9. Brar SS, Provvidenza C, Hunter A, et al.: Improving multidisciplinary cancer conferences: a population-based intervention. Ann Surg Oncol. 2014, 21:16-21. 10.1245/s10434-013-3296-8

10. El Saghir NS, Keating NL, Carlson RW, Khoury KE, Fallowfield L: Tumor boards: optimizing the structure and improving efficiency of multidisciplinary management of patients with cancer worldwide. American Society of Clinical Oncology Educational Book. Dizon DS (ed): American Society of Clinical Oncology, Alexandria, VA; 2014. 34:461-466.

11. Sundi D, Cohen JE, Cole AP, et al.: Establishment of a new prostate cancer multidisciplinary clinic: format and initial experience. Prostate. 2015, 75:191-199. 10.1002/pros.22904

12. Kelly SL, Jackson JE, Hickey BE, Szallasi FG, Bond CA: Multidisciplinary clinic care improves adherence to best practice in head and neck cancer. Am J Otolaryngol. 2013, 34:57-60. 10.1016/j.amjoto.2012.08.010

13. Vinod SK, Sidhom MA, Gabriel GS, Lee MT, Delaney GP: Why do some lung cancer patients receive no anticancer treatment?. J Thorac Oncol. 2010, 5:1025-1032.

10.1097/JTO.0b013e3181da85e4

14. van Hoeve J, de Munck L, Otter R, de Vries J, Siesling S: Quality improvement by implementing an integrated oncological care pathway for breast cancer patients. Breast. 2014, 23:364-370. 10.1016/j.breast.2014.01.008

15. Newman EA, Guest AB, Helvie MA, et al.: Changes in surgical management resulting from case review at a breast cancer multidisciplinary tumor board. Cancer. 2006, 107:2346-2351. $10.1002 /$ cncr.22266

16. Mihalik JE, Krupka L, Davenport R, Tucker L, Toevs C, Smith RS: The rate of imaginghistologic discordance of benign breast disease: a multidisciplinary approach to the management of discordance at a large university-based hospital. Am J Surg. 2010, 199:319323. 10.1016/j.amjsurg.2009.11.004

17. Kesson EM, Allardice GM, George WD, Burns HJ, Morrison DS: Effects of multidisciplinary team working on breast cancer survival: retrospective, comparative, interventional cohort study of 13722 women. BMJ. 2012, 344:e2718. Accessed: October 28, 2016: http://www.bmj.com/content/344/bmj.e2718. 10.1136/bmj.e2718

18. Forrest LM, McMillan DC, McArdle CS, Dunlop DJ: An evaluation of the impact of a multidisciplinary team, in a single centre, on treatment and survival in patients with inoperable non-small-cell lung cancer. Br J Cancer. 2005, 93:977-978. 10.1038/sj.bjc.6602825

19. Yopp AC, Mansour JC, Beg MS, et al.: Establishment of a multidisciplinary hepatocellular carcinoma clinic is associated with improved clinical outcome. Ann Surg Oncol. 2014, 21:1287-1295. 10.1245/s10434-013-3413-8

20. Lordan JT, Karanjia ND, Quiney N, Fawcett WJ, Worthington TR: A 10-year study of outcome following hepatic resection for colorectal liver metastases - The effect of evaluation in a multidisciplinary team setting. Eur J Surg Oncol. 2009, 35:302-306.

10.1016/j.ejso.2008.01.028

21. Stephens MR, Lewis WG, Brewster AE, et al.: Multidisciplinary team management is associated with improved outcomes after surgery for esophageal cancer. Dis Esophagus. 2006, 19:164-171. 10.1111/j.1442-2050.2006.00559.x

22. Munro A, Brown M, Niblock P, Steele R, Carey F: Do multidisciplinary team (MDT) processes 


\section{Cureus}

influence survival in patients with colorectal cancer? A population-based experience. BMC Cancer. 2015, 15:686. Accessed: October 28, 2016:

http://bmccancer.biomedcentral.com/articles/10.1186/s12885-015-1683-1. 10.1186/s12885015-1683-1

23. Look Hong NJ, Gagliardi AR, Bronskill SE, Paszat LF, Wright FC: Multidisciplinary cancer conferences: exploring obstacles and facilitators to their implementation. J Oncol Pract. 2010, 6:61-68. 10.1200/JOP.091085

24. Chirgwin J, Craike M, Gray C, Watty K, Mileshkin L, Livingston PM: Does multidisciplinary care enhance the management of advanced breast cancer?: evaluation of advanced breast cancer multidisciplinary team meetings. J Oncol Pract. 2010, 6:294-300.

10.1200/JOP.2010.000017

25. Jalil R, Ahmed M, Green JS, Sevdalis N: Factors that can make an impact on decision-making and decision implementation in cancer multidisciplinary teams: an interview study of the provider perspective. Int J Surg. 2013, 11:389-394. 10.1016/j.ijsu.2013.02.026

26. Sheehan D, Robertson L, Ormond T: Comparison of language used and patterns of communication in interprofessional and multidisciplinary teams. J Interprof Care. 2007, 21:17-30. 10.1080/13561820601025336

27. Ruhstaller T, Roe H, Thürlimann B, Nicoll JJ: The multidisciplinary meeting: An indispensable aid to communication between different specialities. Eur J Cancer. 2006, 42:2459-2462. 10.1016/j.ejca.2006.03.034 